PACS 32.80.RM; 05.45.+B;

УДК 539.142, 539.184

\title{
SENSING THE CAPTURE OF NEGATIVE MUON BY ATOMS: ENERGY APPROACH
}

\author{
A. V. Glushkov ${ }^{1,2}$, O. Yu. Khetselius ${ }^{1}$, Yu. V. Dubrovskaya ${ }^{2}$, A. V. Loboda \\ ${ }^{1}$ Odessa National Polytechnical University, Odessa, Ukraine \\ ${ }^{2}$ Odessa State Environmental University, Odessa, Ukraine \\ glushkov@paco.net
}

\begin{abstract}
SENSING THE CAPTURE OF NEGATIVE MUON BY ATOMS: ENERGY APPROACH

A. V. Glushkov, O. Yu. Khetselius, Yu. V. Dubrovskaya, A. V. Loboda

It is presented a new method to sensing and estimating the cross-section of the negative muon capture by atoms within a consistent energy approach in the QED theory. Estimates of cross-section of the $\mu^{-}$capture by $\mathrm{He}$ are obtained.
\end{abstract}

Key words: sensing negative muon capture, atomic systems, energy approach

Резюме

\section{ДЕТЕКТУВАННЯ ЗАХОПЛЕННЯ НЕГАТИВНОГО МЮОНА АТОМАМИ: ЕНЕРГЕТИЧНИЙ ПІДХІД}

\section{О. В. Глушков, О. Ю. Хещеліус, Ю. В. Дубровська. А. В. Лобода}

Представлений новий, послідовний підхід до детектування та оцінки процесу захоплення негативного мюону атомами, який базується на енергетичному підході в КЕД теорії. Виконані оцінки перерізів захоплення негативного мюону атомом Не.

Ключові слова: детектування захоплення негативного мюону, атомні системи, енергетичний підхід

Резюме

\section{ДЕТЕКТИРОВАНИЕ ЗАХВАТА ОТРИЦАТЕЛЬНОГО МЮОНА АТОМАМИ: ЭНЕРГЕТИЧЕСКИЙ ПОДХОД}

\section{А. В. Глушков, О. Ю. Хещелиус, Ю. В. Дубровская. А. В. Лобода}

Представлен новый, последовательный подход к детектированию и оценке процесса захвата отрицательного мюона атомами. Выполнены оценки сечений захвата отрицательного мюона атомом Не.

Ключевые слова: детектирование захвата отрицательного мюона, атомные системы, энергетический подход

In last years with appearance of the intensive neutron pensils, powerful laser sources new interest attracts a class of problems, connected with the $\gamma$ quantum-muon-nuclear interactions (e.g.[1-20]).
Speech is about processes of elementary particlenuclear interactions, which were studied ealier, but for significantly other energy and geometry scales. It is well known that a negative muon $\mu$ captures 
by a metastable nucleus may accelerate the discharge of the latter by many orders of magnitude (e.g. $[1,9,16])$. Principal possibility of storage of significant quantities of the metastable nuclei in processes of the nuclear technology and their concentrating by chemical and laser methods leads to question regarding methods of governing velocity of their decay. It had been studied a possibility of action on processes of decay of the nuclei with participating the electrons of atomic shells (K-capture and internal conversion)by means their ionization (e.g. $[1,9,14-16])$. It had been considered a possibility of accelerating discharge of a metastable nucleus by means of the angle momentum part to electron shell of atom $[9,20]$. A comprehensive QED theory of cooperative laser-electron-nuclear processes is developed in refs. $[19,20]$. An effect of electron shell is quite small as the parameter $r_{r} / r_{a}$ is small ( $r_{n}$ is a radius of nucleus and $r_{a}$ is a radius of atom). A meso-atomic system differs advantageously of usual atom, as a relation $r_{n} / r_{a}$ can vary in the wide limits in dependence upon the nuclear charge. For a certain relation between the energy range of the nuclear and muonic levels the discharge of metastable nucleus may be followed by the ejection of a muon, which may then participate in the discharge of other nuclei. Estimates of probabilities for discharge of a nucleus with emission of $\gamma$ quantum and further muon or electron conversion are presented in ref. [16]. Let us also underline that despite the relatively long studying the processes of interaction of mesons with atoms, nuclei hitherto there is no absolutely common opinion regarding values of energies when the muons are captured by different atoms and nuclei. The corresponding experiment is connected with known difficulties, and different theoretical estimates are significantly differ from each other. According to Mann and Rose (e.g.[4]), the muon capture occurs mainly under energies of $\mathrm{E} \sim 10 \mathrm{KeV}$, but according to Bayer muons survive till thermal energies. In majority of papers (e.g. $[1,4])$ different authors predicted the muon capture energies in interval from a few dozens to thoursands $\mathrm{eV}$. Traditional theoretical approaches to problem are based on the Born approximation with plane or disturbed wave functions and the hydrogen-like functions for the discrete states. For estimating the muon energies with the most high capture probability in many papers there is used the cross-section of inelastic scattering of muons on atoms by Rosenberg, which is however significantly decreased. In papers by Vogel et al and Leon-Miller (e.g.[4]) it is used the well known Fermi-Teller model, in which the atomic electrons are treated as an electron gas and muon is classically described. In paper by Cherepkov and Chernysheva [4] the standard Hartree-Fock mathod is used to define the cross-sections of the Auger capture, elastic and inelastic scattering of the negative muons on atom of $\mathrm{He}$ in the energy range from 1 to $175 \mathrm{eV}$. The standard Hartree-Fock wave functions are used for description of atom and removed electron and the Hartree-Fock functions in a field of "freezy" atom and ion respectively are used for treating the muon in the initial and final states. More modern approaches [2], based on the fermion molecular dynamics method began to use in the last years in the scattering and capture problem. In any case we suppose that more sophisticated methods of the QED perturbation theory and an energy approach type (e.g.[12-20]) are needed for correct solving the muon capture not only by light atoms, but by the heavy ones too.

Here we present a new method to sensing and estimating the cross-section of the negative muon capture using a consistent energy approach $[12,14,17]$ in the QED theory. Let us present the key moments of the approach. In the theory of the atom a convenient field procedure is known for calculating the energy shifts $\Delta E$ of degenerate states. This procedure is connected with the secular matrix $M$ diagonalization. In constructing $M$, the Gell-Mann and Low adiabatic formula for $\Delta E$ is used. A similar approach, using the Gell-Mann and Low formula with the QED scattering matrix, is applicable in the relativistic atom theory (c.f.[9-12]). In contrast to the non-relativistic case, the secular matrix elements are already complex in the second order of the perturbation theory (PT) (first order of the inter-electron interaction). Their imaginary parts are connected with the radiation decay (radiation) possibility. The total energy shift of the state is usually presented in the form:

$$
\Delta E=\operatorname{Re} \Delta E+\mathrm{i} \operatorname{Im} \Delta E \quad \operatorname{Im} \Delta E=-\Gamma / 2
$$

where $\Gamma$ is interpreted as the level width, and the decay possibility $P=\Gamma$. The whole calculation of the energies and decay probabilities of a non-degenerate excited state is reduced to calculation and diagonalization of the complex matrix $M$. To start with the QED Gell-Mann and Low formula one must choose the zero-order approximation. Usually one uses for this purpose a one-electron Hamiltonian with a central potential that can be treated as a bare potential in the formally exact QED PT. There are many well-known 
attempts to find the more fundamental optimization principles for the bare one-electron Hamiltonian or (what is the same) for the basis of one-electron functions which represents such a Hamiltonian. The minimization of the gauge dependent multielectron contribution of the lowest QED PT corrections to the radiation widths of atomic levels is proposed in [12] as "ab initio" optimization principle (see below). In present calculations of the muon capture we dealt with atoms and ions having the closed electron shells. For example, the ground state $1 s^{2}$ of the He atom or He-like ion. Note that below we operate in the relativistic approximation, though for light atoms $(\mathrm{H}$ or $\mathrm{He})$ the non-relativistic approach is suitable. It is usually accepted, as the bare potential, a potential including the electric nuclear potential $\mathrm{V}_{\mathrm{N}}$ and some parameterized potential $\mathrm{V}_{\mathrm{C}}$, that imitates the interaction of closed-shell electrons with incident particles. The parameters of the model bare potential are chosen so as to generate accurate eigen-energies of all two-particle (2QP) states, i.e. $1 \mathrm{~s} 2 \mathrm{~s}, 2 \mathrm{p} 3 \mathrm{~s}, 3 \mathrm{p}, 3 \mathrm{~d}$ etc. with the same nucleus. In the second order of the QED PT the energy shift is expressed in terms of two-particle matrix elements

$$
\begin{gathered}
V(1,2 ; 4,3)=\sqrt{\left(2 j_{1}+1\right)\left(2 j_{2}+1\right)\left(2 j_{3}+1\right)\left(2 j_{4}+1\right)} \times \\
\times(-1)^{j_{1}+j_{2}+j_{3}+j_{4}+m_{1}+m_{2}} \times \\
\times \sum_{\lambda, \mu}(-1)^{\mu}\left[\begin{array}{c}
j_{1} \ldots \ldots . j_{3} \ldots \lambda \\
m_{1} \cdot-m_{3 . \ldots} \mu
\end{array}\right]\left[\begin{array}{l}
j_{2} \ldots \ldots . j_{4} \ldots \lambda \\
m_{2} . \cdot m_{4} . . \mu
\end{array}\right] \times \\
\times\left(Q_{\lambda}^{Q u l}+Q_{\lambda}^{B r}\right)
\end{gathered}
$$

Here $Q_{\lambda}^{\text {Qul }}$ is corresponding to the Coulomb inter-particle interaction:

$$
\begin{gathered}
Q_{\lambda}^{\mathrm{Qul}}=\left\{R_{\lambda}(1243) S_{\lambda}(1243)+\right. \\
\left.\left.+R_{\lambda} \tilde{1} 24 \tilde{3}\right) S_{\lambda} \tilde{1} 24 \tilde{3}\right)+R_{\lambda}(\tilde{2} \tilde{4} 3) S_{\lambda}(1 \tilde{2} \tilde{4} 3) \\
\left.\left.\left.+R_{\lambda} \tilde{1} \tilde{2} \tilde{4} \tilde{3}\right) S_{\lambda} \tilde{(} \tilde{2} \tilde{4} \tilde{3}\right)\right\}
\end{gathered}
$$

where $R_{\lambda}(1,2 ; 4,3)$ is the radial integral of the Coulomb inter-electron interaction with large radial components; the tilde denotes a small component; $\mathrm{S}$ is the angular multiplier (see expressions in ref.[18,19]). Expession for the Breit inter-particle interaction part is similar to (3) and presented also in ref.[16-19]. To calculate all necessary matrix elements one must use the basis's of the 1QP relativistic functions. In many calculations of characteristics of the atomic elementary processes it has been shown that adequate description of these characteristics requires using the optimized basis's of wave functions. In ref. [12] it has been proposed "ab initio" optimization principle for construction of cited basis's. There is used the minimization of the gauge dependent multielectron contribution of the lowest QED PT corrections to the radiation widths of atomic levels. The details of procedure can be found in [12]. Below we first use such a basis in calculation of the muon capture cross-section. Further we briefly discuss the energy approach in scattering theory 11,12] and give main formulas. We briefly outline the main idea using, as an example, the negative muon capture by $\mathrm{He}$ atom: $\left((1 s)^{2}\left[J_{i} M_{i}\right]\right.$, $\left.\varepsilon_{i n}{ }^{\mu}\right) \rightarrow\left(1 s \varepsilon l, \varepsilon_{n l}{ }^{\mu}\right)$. Here $J_{i}$ is the total angular moment of the initial target state; indices $\varepsilon_{i n}{ }^{\mu}$ and $\varepsilon_{f k}{ }^{\mu}$ are the incident and discrete state energies, respectively to the incident and captured muons. Further it is convenient to use the second quantization representation. In particular, the initial state of the system "atom plus free muon" can be written as $a_{i n}^{+\mu} \Phi_{0}$ state. Final state is the state of an atom with the discrete state electron, removed electron and captured muon; in furhter $|I\rangle$ represents one-particle (1QP) state , and $|F\rangle$ represents the three-quasiparticle (3QP) state. The Auger capture part of energy shift Im $\Delta E$ appears first in the second order of the atomic perturbation theory (fourth order of the QED perturbation theory) in the form:

$$
\operatorname{Im} \Delta E=\pi G\left(\varepsilon_{i v}, \varepsilon_{i e}, \varepsilon_{i n}^{\mu}, \varepsilon_{f k}^{\mu}\right)
$$

Indices $e, v$ are coresponding to atomic electrons. Here $G$ is a definite squired combination of the twoparticle matrix elements (2). The value $\sigma=-2 \operatorname{Im} \Delta E$ represents the capture cross-section if the incident muon eigen-function is normalized by the unit flow condition. The cross-section is as follows:

$$
\begin{gathered}
\sigma(0 \rightarrow I F)=2 \pi\left(2 J_{f}+1\right) \sum_{j_{i n}, j_{s c}}\left(2 j_{s c}+1\right) \cdot \\
\left\{\sum_{j_{f e}, j_{j v}} B_{f e, f v}^{F K}<j_{f e}, j_{f v} J_{f}\left|j_{i n}, j_{s c}\right| 0>\right\}^{2}
\end{gathered}
$$

Here $B_{i e, i v}^{I K}$ is a real matrix of eigen-vectors coefficients, which is obtained after diagonalization of the secular energy matrix. The amplitude like combination in (8) has the following form:

$$
\begin{gathered}
<0\left|j_{i n}^{\mu}, j_{f k}^{\mu}\right| j_{i e}, j_{i v}, J_{i}>= \\
=\sqrt{\left(2 j_{i e}+1\right)\left(2 j_{i v}+1\right)}(-1)^{j_{i e}+1 / 2} \times \sum_{\lambda}(-1)^{\lambda+J_{i}} \times \\
\times\left\{\delta_{\lambda, J_{i}} /\left(2 J_{i}+1\right) Q_{\lambda}(f k, i e ; i v, i n)+\right. \\
\left.+\left[\begin{array}{c}
j_{i n} \ldots j_{f k} \ldots J_{i} \\
j_{i e} \ldots j_{i v} \ldots . . \lambda
\end{array}\right] Q_{\lambda}(i e ; i n ; i v, f k)\right\}
\end{gathered}
$$


In (15) values $Q_{\lambda}^{\mathrm{Qul}}$ and $Q_{\lambda}^{\mathrm{Br}}$ are defined by the expressions of the type (3). The different normalization conditions are used for the incident and for the captured state electron wave functions. The details of the whole numerical procedure of calculation of the capture cross-section and also elastic and inelastic cross-sections can be found in $[16,17]$.

We have carried out the calculation of the negative muon capture by atom of He. At first we studied the behaviour of the curves for cross-section of capture for the negative muons in dependence upon the ground quantum number $n$ with summation on the orbital moments $l$ for several values of hte muon initial energy. In whole our curves are lying a little highe than ones [4]. Analysis shows that the capture cross-section for energies of the incident muon 16 and $50 \mathrm{eV}$ with growth of the orbital quantum number $(l>10)$ begins to decrease for all $n$. For small and large energies of muons in comparison with atomic ionization potential) states with large $l$ are populated with less probability than for energies of the ionization potential order. It is qualitatively similar to the non-relativistic Hartree-Fock calculation. The more probable capture occurs to states with $n=15-20$. At the same time the HartreeFock basises are not gauge-invariant ones. So, one could under-stand that an accuracy of calculation with the gauge-invariant basises within an energy approach is naturally higher. Calculated in present paper full cross-sections of the muon capture, elastic and inelastic scattering in dependence upon the energy with summation on all $n, l$ are presented in fig 1 (dotted line curves 7,8,9 respectively in fig.). For comparison we present the cross-sections of the Auger capture, elastic and inelastic scattering, obtained within the Hartree-Fock method (curves $1,2,3)$, cross-section of the inelastic scattering by Rosenberg (curve 4), cross-section of the Auger capture by Copenman and Rogova (curve 5) and so called transport cross-section $[1,4]$.

The Hartree-Fock basises are not gauge-invariant ones. So, one could understand that an accuracy of calculation with the gauge-invariant basises within consistent energy approach (present paper) is naturally higher. This is regarding data by Rosenberg, Copenman-Rogova and transport cross- sections. More detailed discussion regarding an importance of using the optimized gauge-invariant basises in the cross-section calculation and quantitative estimates are given in refs.[16-20]. In any case, we can conclude that a new proposed method to sensing and estimating the cross-section of the negative muon capture by atoms within a consistent energy approach in the QED theory can be usefully used in solving problem considered, in particular, in calculation of the Auger-capture of negative muons by heavy relativistic atoms.

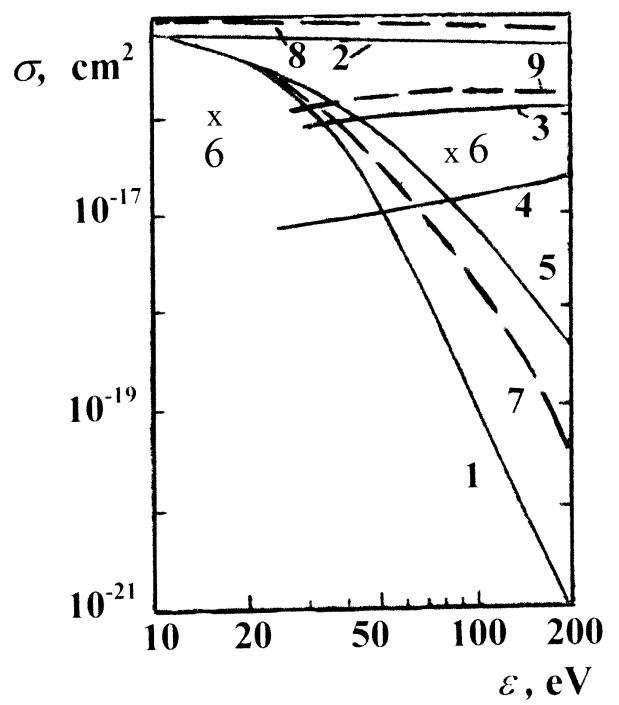

Fig. 1. The cross-section of the Auger capture, elastic and inelastic scattering, obtained within the HartreeFock method (curves 1,2,3), cross-section of the inelastic scattering by Rosenberg (curve 4), cross-section of the Auger capture by Copenman and Rogova (curve 5), the transport cross-section (cross symbol) $[1,4]$ and the muon capture, elastic and inelastic scattering (present paper; dotted line curves $7,8,9$ respectively).

\section{Acknowledgements}

Author would like to thank Profs. W.Kohn, E.Brдndas, L.Ivanov, I.Kaplan, J.Maruani, C.Roothan, A.Theophilou for useful discussion.

\section{References}

1. Eds. Aumar F. and Winter H. Photonic, Electronic and Atomic Collisions. - Singapore: World Sci. 1997.

2. Cohen J.S., Capture of antiprotons by some radioactive atoms and ions//Phys.Rev.A. - 2004. Vol.69. - P.022501 1-10.

3. Kravtsov A.V., Mikhailov A.I., Muon transfer from excited muonic hydrogen to helium// Phys.Rev.A. 1994. - Vol.49. - P.3566-3582.

4. Cherepkov N.A., Chernysheva L.V., Capture of negative mesons by atoms of $\mathrm{He} / / \mathrm{Journ}$. of Nucl. Phys. - 1980. - vol.32. - P.709-718.

5. Glushkov A.V., Malinovskaya S.V., Co-operative laser nuclear processes: border lines effects// In: New projects and new lines of research in nuclear physics. 
Eds. G.Fazio and F.Hanappe, Singapore : World Scientific. - 2003. - P.242-250.

6. Glushkov A.V., Gurnitskaya E.P., Loboda A.V., Advanced quantum mechanical calculation of superheavy ions: energy levels, Radiation and Finite $\mathrm{Nu}-$ clear size effects// Low Energy Antiproton Phys., AIP Serie. - 2005. - Vol.796. - P.217-220.

7. Harston M.R., Carroll J.J., Nuclear excitation and de-excitation in resonant electronic transitions// Laser Phys. - 2004. - Vol.14. - P.1452-1463

8. Wauters L., Vaeck, N. Study of the electronic rearrangement induced by nuclear transmutations: A Bspline approach applied to the beta decay of ${ }^{6} \mathrm{He} / /$ Phys.Rev.C. - 1996. - Vol.53. - P.497-502..

9. Goldansky V.I., Letokhov V.S. Effect of laser radiation on nuclear decay processes// Sov. Phys. JETP. 1974. - Vol.67. - P.513-516.

10. Ivanov L.N., Letokhov V.S. Possibility of discharge of metastable nuclei during negative muon capture// Sov.Phys. JETP. - 1976. - Vol.71-P.19-28.

11. Ivanov L.N., Letokhov V.S. Spectroscopy of autoionization resonances in heavy elements atoms// Com. Mod.Phys.D.:At.Mol.Phys. - 1985. - Vol.4. P.169-184.

12. Glushkov A.V., Ivanov L.N. Radiation Decay of Atomic States: atomic residue and gauge non-invariant contributions // Phys. Lett.A. - 1992. Vol.170,N1. - P.33-37.

13. Glushkov A.V. Negative Ions of inert Gases// Pis'ma to JETP. - 1992. — Vol.55- P.104-107; JETP Lett. 1992. - Vol.55. - P.97-100

14. Glushkov A.V., Energy Approach to Resonance states of compound super-heavy nucleus and EPPP in heavy nucleus collisions// Low Energy Antiproton Phys., AIP Serie. - 2005. - Vol.796. - P.206-210
15. Glushkov A.V., Rusov V.D., Ambrosov S.V., Loboda A.V. Resonance states of compound super-heavy nucleus and EPPP in heavy nucleus collisions // In: New projects and new lines of research in nuclear physics.Eds. G.Fazio, F.Hanappe, Singapore : World Scientific. - 2003. - P.126-132.

16. Glushkov A.V., Malinovskaya S.V., Dubrovskaya Yu.V., Vitavetskaya L.A., Quantum calculation of cooperative muon-nuclear processes: discharge of metastable nuclei during negative muon capture// Recent Advances in Theor. Phys. and Chem. Systems. - 2006. - Vol.15. - P.301-308.

17. Glushkov A.V., Malinovskaya S.V., Gurnitskaya E.P., Khetselius O.Yu.,Dubrovskaya Yu.V., Consistent quantum theory of the recoil induced excitation and ionization in atoms during capture of neitron// J.Phys.CS. - 2006. - Vol.35. - P.425-430.

18. Glushkov A.V., Ambrosov S.V., Loboda A.V., Gurnitskaya E.P., Prepelitsa G.P., Consistent QED approach to calculation of electron-collision excitation cross-sections and strengths: Ne-like ions // Int. Journ.Quant.Chem. - 2005. - Vol.104, N4. P. 562-569.

19. Glushkov A.V., Malinovskaya S.V., Loboda A.V., Shpinareva I.M., Gurnitskaya E.P., Korchevsky D.A., Diagnostics of the collisionally pumped plasma and search of the optimal plasma parameters of $\mathrm{x}$-ray lasing: Calculation of electron-collision strengths and rate coefficients for Ne-like plasma// J.Phys.CS. 2005. - Vol.178. - P.188-198

20. Glushkov A.V., Malinovskaya S.V., Svinarenko A.A., Chernyakova Yu.G., QED Calculation of Electron Satellites Spectra in Intense Laser Field in Multicharged Ion//Int.J.Quant.Chem. - 2004. Vol.99. - P.889-896. 